Vietnam Journal of Mechanics, VAST, Vol.40, No. 3 (2018), pp. $251-264$

DOI: https://doi.org/10.15625/0866-7136/11085

\title{
PARAMETRIC CONDITIONS AND EXACT SOLUTION FOR THE DUFFING-VAN DER POL CLASS OF EQUATIONS
}

\author{
Dao Huy Bich ${ }^{1}$, Nguyen Dang Bich ${ }^{2, *}$ \\ ${ }^{1}$ VNU Hanoi University of Science, Hanoi, Vietnam \\ ${ }^{2}$ Institute for Buiding Science and Technology, Hanoi, Vietnam \\ *E-mail: dangbichnguyen@gmail.com \\ Received January 06, 2018
}

\begin{abstract}
This paper presents a methodology to find the exact solution and respective parametric conditions to the Duffing-Van der Pol class of equations. The supposed method in this paper is different from the Prelle and Singer method and the Lie symmetry method. The main idea of the supposed method is implemented in finding the first integrals of the original equation and leading this equation to a solved equation of lower order to which the exact solution can be obtained. As results the parametric conditions and the exact solutions in parametric forms are indicated. The algorithm for determining integral constants and the investigation of solution characteristics are considered.
\end{abstract}

Keywords: parametric conditions, Prelle and Singer method, Lie symmetry method, exact solutions, Duffing-Van der Pol equation, Riccati equation, hypergeometric functions.

\section{INTRODUCTION}

Problems in mechanics, physics, chemistry and biology often lead to nonlinear differential equations. Finding new solutions to classes of differential equations is always an interest to scientists in the field of dynamics. For this purpose a first integral should be found, based on which the exact solution is obtained. The closed form structure of the solution is an important basis for observations made on mechanics, physics, chemistry and biology phenomena.

Pioneered by Prelle and Singer [1], the methodology for finding the first integral of a first order ordinary differential equations was introduced. Duarte et al. [2] and Chandrasekar et al. [3] extended this methodology to second order ordinary differential equations. The symmetry Lie method can be used for the examination of the nonlinear dissipative system. Senthil et al. [4] and Chandrasekar et al. [5] used the symmetry Lie method to solve for the first integral of a force-free Duffing-Van der Pol equation. Other scientists [6-8] have examined different classes of nonlinear differential equations and contributed methodology for finding the corresponding first integral and exact solution.

(C) 2018 Vietnam Academy of Science and Technology 
The Duffing-Van der Pol class of nonlinear differential equation is examined in this paper

$$
\ddot{x}+\left(\delta+\beta x^{m}\right) \dot{x}-\mu x+x^{m+1}=0,
$$

where an overdot represents differentiation with respect to independent variable $t$. All other parameters such as $\delta, \beta$ and $\mu$ are real numbers, and $\mathrm{m}$ is another number of any value.

This paper proposes a methodology to find the first integral and exact solution to the above equation in a different approach in comparison to the existing Prelle and Singer [1] and the symmetry Lie methods. Not only that the proposed methodology renders an identical solution in comparison with results from the symmetry Lie method when $m=2$, but it can also provide a general solution and new observations to the DuffingVan der Pol equation with any $m$.

\section{PARAMETRIC CONDITIONS AND FIRST INTEGRALS}

Let us consider the Duffing-Van der Pol type oscillator equation (1) with arbitrary power $m$. With $m=2$, Eq. (1) has of the form of the classic Duffing-Van der Pol equation

$$
\ddot{x}+\left(\delta+\beta x^{2}\right) \dot{x}-\mu x+x^{3}=0,
$$

and then $\beta=0$ this equation leads to the damped Duffing equation

$$
\ddot{x}+\delta \dot{x}-\mu x+x^{3}=0 .
$$

For finding first integrals of Eq. (1) we use the transformations [9] as follows

$$
\begin{aligned}
& \frac{\dot{x}}{x}+\frac{1}{2} a x^{m}+\frac{1}{2}(b+c)=\frac{\dot{u}}{u}, \\
& -\frac{1}{2} a x^{m}+\frac{1}{2}(b-c)=\frac{1}{2} \frac{\dot{v}}{v},
\end{aligned}
$$

which lead Eq. (1) to the following equation

$$
\frac{\mathrm{d}}{\mathrm{d} t}\left(\frac{\dot{u}}{u}\right)+\left(\frac{\dot{u}}{u}\right)^{2}+\frac{\mathrm{d}}{\mathrm{d} t}\left(\frac{1}{2} \frac{\dot{v}}{v}\right)-\left(\frac{1}{2} \frac{\dot{v}}{v}\right)^{2}=0 .
$$

In order to get Eq. (4) be equivalent to Eq. (1) the coefficients in Eqs. (2) and (3) must be relative as

$$
b+c=\delta, \quad a=\beta, \quad a b=1, \quad b c=-\mu .
$$

The system of Eqs. (5) consists of four equations with three unknowns, certainly there exists a relative condition within coefficients. Solving Eqs. (5) yields

$$
a=\beta, \quad b=\frac{1}{\beta}, \quad c=\delta-\frac{1}{\beta}
$$

and

$$
\delta=\frac{1}{\beta}-\mu \beta
$$


Accoding to [9] Eq. (4) has a first integral

$$
\frac{u}{v^{1 / 2}}\left(\frac{\dot{u}}{u}+\frac{1}{2} \frac{\dot{v}}{v}\right)=D_{1}
$$

where $D_{1}$ is an integral constant.

From Eqs. (2) and (3) we obtain

$$
\begin{gathered}
\frac{\dot{x}}{x}+b=\frac{\dot{u}}{u}+\frac{1}{2} \frac{\dot{v}}{v}, \\
\frac{u}{v^{1 / 2}}=x \exp \left[\int_{0}^{t}\left(a x^{m}+c\right) \mathrm{d} t\right] .
\end{gathered}
$$

Substituting Eqs. (9) and (10) into Eq. (8) yields

$$
\dot{x}+b x=D_{1} \exp \left[-\int_{0}^{t}\left(a x^{m}+c\right) \mathrm{d} t\right] .
$$

Consequently, determining $a, b$ and $c$ by Eq. (6) and considering condition (7), we obtain the first integral (11) of the original equation (1). The equality (7) is considered as the parametric condition under which Eq. (1) has a first integral (11).

Now we can show that under another parametric condition, Eq. (1) has other first integral. Multiplying both sides of Eq. (11) by $x^{m}$ yields

$$
x^{m} \dot{x}+b x^{m+1}=D_{1} x^{m} \exp \left[-\int_{0}^{t}\left(a x^{m}+c\right) \mathrm{d} t\right]
$$

or $\frac{\mathrm{d}}{\mathrm{d} t}\left(x^{m+1}\right)+b(m+1) x^{m+1}=D_{1}(m+1) e^{-c t} x^{m} \exp \left[-\int_{0}^{t} a x^{m} \mathrm{~d} t\right]$,

from which we obtain

$$
x^{m+1}=e^{-b(m+1) t}\left\{D_{1}(m+1)\left[\int e^{[b(m+1)-c] t} x^{m} \exp \left(\int_{0}^{t} a x^{m} \mathrm{~d} t\right) \mathrm{d} t\right]+D_{2}\right\},
$$

where $D_{2}$ is an integral constant.

Suppose that parameters satisfy the relation

$$
b(m+1)=c,
$$

using Eq. (6) this relation leads to

$$
m=\delta \beta-2
$$

The integral in the right-hand side of Eq. (12) can be performed and Eq. (12) becomes

$$
x^{m+1}=e^{-c t}\left\{D_{2}-\frac{m+1}{a} D_{1} \exp \left[-\int_{0}^{t} a x^{m} \mathrm{~d} t\right]\right\} \text {. }
$$


In the results, under two parametric conditions (7) and (13) the original equation (1) has another first integral (14) including two integral constants.

Rewriting Eq. (14) as

$$
x^{m+1}=D_{2} e^{-c t}-\frac{m+1}{a} D_{1} \exp \left[-\int_{0}^{t}\left(a x^{m}+c\right) \mathrm{d} t\right],
$$

and combining it with Eq. (11) we obtain the first integral in another form

$$
\dot{x}+b x+\frac{a}{m+1} x^{m+1}=\frac{a D_{2}}{m+1} e^{-c t} .
$$

It is remarkable that the obtained first integral (15) has the form coinciding with the first integral to be found in Ref. [8, p. 129] by another method.

In particular case $m=2$, two parametric conditions (7) and (13) become

$$
\delta=\frac{4}{\beta}, \quad \mu=-\frac{3}{\beta^{2}},
$$

and the first integral (15) has of the form

$$
\dot{x}+\frac{1}{\beta} x+\frac{\beta}{3} x^{3}=\frac{\beta}{3} D_{2} e^{-\frac{3}{\beta} t} .
$$

The parametric conditions (16) and the first integral (17) to be found in this paper fully coincide with those given by Lie symmetry method in Refs. [3, p. 2467], [4, p. 1936] and [5, p. 4528].

\section{SOLVING EQUATION AND EXACT SOLUTION}

Basing on the first integral (14) we derive the solving equation. Introduce a variable transformation

$$
\xi=\exp \left(-\int_{0}^{t} a x^{m} \mathrm{~d} t\right)
$$

from which to get

$$
x^{m}=-\frac{1}{a} \frac{\dot{\xi}}{\bar{\xi}}
$$

Substituting Eqs. (18) and (19) into Eq. (14) yields

$$
\left(-\frac{1}{a} \frac{\dot{\xi}}{\xi}\right)^{\frac{m+1}{m}}=\left(D_{2}-\frac{m+1}{a} D_{1} \xi\right) e^{-c t},
$$

and then arising both sides of just obtained equation by power $\frac{m}{m+1}$ lead to

$$
-\frac{1}{a} \frac{\dot{\xi}}{\xi}=\left(D_{2}-\frac{m+1}{a} D_{1} \xi\right)^{\frac{m}{m+1}} e^{-\frac{c m}{m+1} t}
$$


Eq. (20) can be rewirtten in another form

$$
-\frac{1}{a} \frac{\mathrm{d} \xi}{\xi\left(D_{2}-\frac{m+1}{a} D_{1} \xi\right)^{\frac{m}{m+1}}}=e^{-\frac{c m}{m+1} t} \mathrm{~d} t
$$

Integrating both sides of Eq. (21) yields

$$
\frac{c m}{a(m+1)} \int \frac{\mathrm{d} \xi}{\xi\left(D_{2}-\frac{m+1}{a} D_{1} \xi\right)^{\frac{m}{m+1}}}+\gamma=e^{-\frac{c m}{m+1} t}
$$

where $\gamma$ is an integral constant.

The integral in left-hand side of Eq. (22) can be performed by using an appropriate transformation

$$
z=\left(D_{2}-\frac{m+1}{a} D_{1} \xi\right)^{\frac{1}{m+1}}
$$

A direct calculation gives

$$
\begin{gathered}
\xi=-\left(z^{m+1}-D_{2}\right) \frac{a}{(m+1) D_{1}}, \\
\mathrm{~d} \xi=-\frac{a}{D_{1}} z^{m} \mathrm{~d} z .
\end{gathered}
$$

Substituting the above equalities into Eq. (22) yields

$$
\frac{c m}{a} \int \frac{\mathrm{d} z}{z^{m+1}-D_{2}}+\gamma=e^{-\frac{c m}{m+1} t}
$$

The integral in Eq. (24) can be performed through hypergeometric functions [10], then Eq. (24) can be demonstrated as

$$
\frac{c m}{a}\left\{-\frac{z}{D_{2}} \text { Hypergeometric } 2 F 1\left[\frac{1}{m+1}, 1 ; 1+\frac{1}{m+1} ; \frac{z^{m+1}}{D_{2}}\right]\right\}+\gamma=e^{-\frac{c m}{m+1} t} .
$$

By making the natural logarithm to Eq. (25) yields

$$
t=-\frac{m+1}{c m} \ln \left\{\frac{c m}{a}\left[-\frac{z}{D_{2}} \text { Hypergeometric } 2 F 1\left[\frac{1}{m+1}, 1 ; 1+\frac{1}{m+1} ; \frac{z^{m+1}}{D_{2}}\right]\right]+\gamma\right\} \text {. }
$$

Otherwise, from Eqs. (20) and (23) we obtain

$$
-\frac{1}{a} \frac{\dot{\xi}}{\xi}=z^{m} e^{-\frac{c m}{m+1} t}
$$

by use of Eq. (19) the relation within $x$ and $z$ is given

$$
x=z e^{-\frac{c}{m+1} t},
$$

and Eq. (25) can be rewritten as follows

$$
x=z\left\{\frac{c m}{a}\left[-\frac{z}{D_{2}} \text { Hypergeometric } 2 F 1\left[\frac{1}{m+1}, 1 ; 1+\frac{1}{m+1} ; \frac{z^{m+1}}{D_{2}}\right]\right]+\gamma\right\}^{\frac{1}{m}} .
$$


Eqs. (26) and (28) can be seen as an exact solution demonstrated in parametric form with respect to $z$ of the original equation (1), under parametric conditions (7) and (13).

Now we can get parametric solution for velocity $\dot{x}$ and the phase curve of Eq. (1). For this season taking differentiation with respect to $t$ Eqs. (24) and (27)

$$
\begin{gathered}
\dot{z}=-\frac{a}{m+1}\left(z^{m+1}-D_{2}\right) e^{-\frac{c m}{m+1} t}, \\
\dot{x}=\dot{z} e^{-\frac{c}{m+1} t}-\frac{c}{m+1} z e^{-\frac{c}{m+1} t},
\end{gathered}
$$

and using just obtained equalities and Eq. (25) we obtain

$$
\begin{aligned}
& \dot{x}=-\frac{c}{m+1} z\left\{\frac{c m}{a}\left[-\frac{z}{D_{2}} \text { Hypergeometric } 2 F 1\left[\frac{1}{m+1}, 1 ; 1+\frac{1}{m+1} ; \frac{z^{m+1}}{D_{2}}\right]\right]+\gamma\right\}^{\frac{1}{m}} \\
& -\frac{a}{m+1}\left(z^{m+1}-D_{2}\right)\left\{\frac{c m}{a}\left[-\frac{z}{D_{2}} \text { Hypergeometric } 2 F 1\left[\frac{1}{m+1}, 1 ; 1+\frac{1}{m+1} ; \frac{z^{m+1}}{D_{2}}\right]\right]+\gamma\right\}^{\frac{m+1}{m}} .
\end{aligned}
$$

Finally, the overall results can be drawn: suppose the Duffing-Van der Pol type oscillator equation is given in the form of Eq. (1). If its parameters satisfy relative conditions (7) and (13)

$$
\begin{aligned}
& \delta=\frac{1}{\beta}-\mu \beta, \\
& m=\delta \beta-2,
\end{aligned}
$$

then the given equation has an exact solution in parametric form represented by Eqs. (26) and (28), a velocity - by Eqs. (26) and (29) and a phase curve - by Eqs. (28) and (29). Parameters $a$ and $c$ in the mentioned above equations are defined by relations (6)

$$
a=\beta, \quad b=\frac{1}{\beta}, \quad c=\delta-\frac{1}{\beta} .
$$

\section{DETERMINATION OF INTEGRAL CONSTANTS}

Suppose the initial conditions are given as

$$
\begin{aligned}
& \left.x(t)\right|_{t=0}=x_{0}, \\
& \left.\dot{x}(t)\right|_{t=0}=\dot{x}_{0},
\end{aligned}
$$

from Eq. (27) it leads to

$$
\left.z(t)\right|_{t=0}=x_{0} .
$$

Based on Eqs. (26) and (30) the integral constant $\gamma$ is defined

$$
\gamma=1-\frac{c m}{a}\left\{-\frac{x_{0}}{D_{2}} \text { Hypergeometric } 2 F 1\left[\frac{1}{m+1}, 1 ; 1+\frac{1}{m+1} ; \frac{x_{0}^{m+1}}{D_{2}}\right]\right\},
$$

and the integral constant $D_{2}$ is found by the first integral (15)

$$
D_{2}=\frac{m+1}{a} \dot{x}_{0}+\frac{c}{a} x_{0}+x_{0}^{m+1} .
$$




\section{APPLICATIONS}

In this section some cases of Eq. (1) are considered and numerical cases are given

\subsection{Case $m=1$}

Parametric conditions (7) and (13) in this case are

$$
\delta=\frac{3}{\beta}, \quad \mu=-\frac{2}{\beta^{2}},
$$

the original equation (1) has the form

$$
\ddot{x}+\left(\frac{3}{\beta}+\beta x\right) \dot{x}+\frac{2}{\beta^{2}} x+x^{2}=0,
$$

and according to Eq. (15) there exists a first integral

$$
\dot{x}+\frac{1}{\beta} x+\frac{\beta}{2} x^{2}=\frac{\beta D_{2}}{2} e^{-\frac{2}{\beta} t} .
$$

This equation has the form of Riccati equation.

The exact solution of the given equation is obtained from Eq. (26) with $m=1, a=\beta$ and $c=\frac{2}{\beta}$

$$
x=\sqrt{D_{2}} e^{-\frac{1}{\beta} t} t h\left(-\frac{\beta^{2}}{2} \sqrt{D_{2}} e^{-\frac{1}{\beta} t}+\gamma\right),
$$

where

$$
\begin{aligned}
& \gamma=\operatorname{arth}\left(\frac{x_{0}}{\sqrt{D_{2}}}\right)+\frac{\beta^{2}}{2} \sqrt{D_{2}}, \\
& D_{2}=\frac{2}{\beta} \dot{x}_{0}+\frac{2}{\beta^{2}} x_{0}+x_{0}^{2} .
\end{aligned}
$$

Suppose that the input data are given in some cases.

(i) $\beta=2.5$ and initial values $x_{0}=1, \dot{x}_{0}=0.4$, integral constants are calculated by Eqs. (31) and (32)

$$
D_{2}=1.64, \gamma=5.04955
$$

The exact solution is represented in Fig. 1.

(ii) $\beta=2.5$ and initial values $x_{0}=1, \dot{x}_{0}=-2$, integral constants are valued as

$$
D_{2}=-0.28, \gamma=0.569439 i \text {. }
$$

The exact solution is illustrated in Fig.2. follows

(iii) $\beta=2.5$ and initial values $x_{0}=1, \dot{x}_{0}=-3$, integral constants are calculated as

$$
D_{2}=-1.08, \gamma=2.48143 i \text {. }
$$

The exact solution is shown in Fig. 3 , it has an asymptotic line $t \approx 3.2$. 


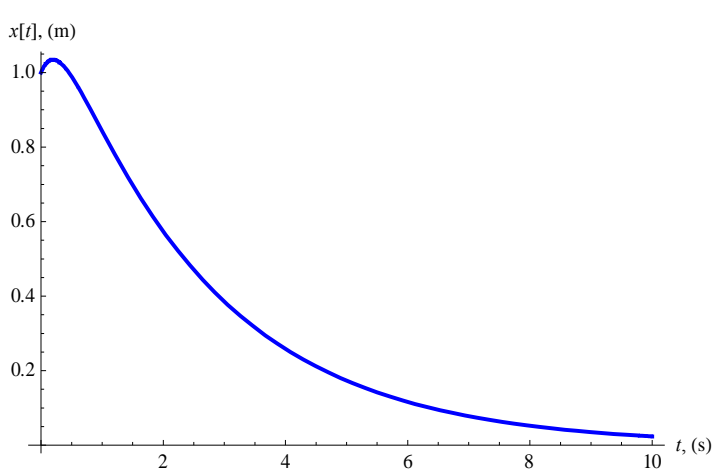

Fig. 1. The exact solution with $\beta=2.5, x_{0}=1$ and $\dot{x}_{0}=0.4$

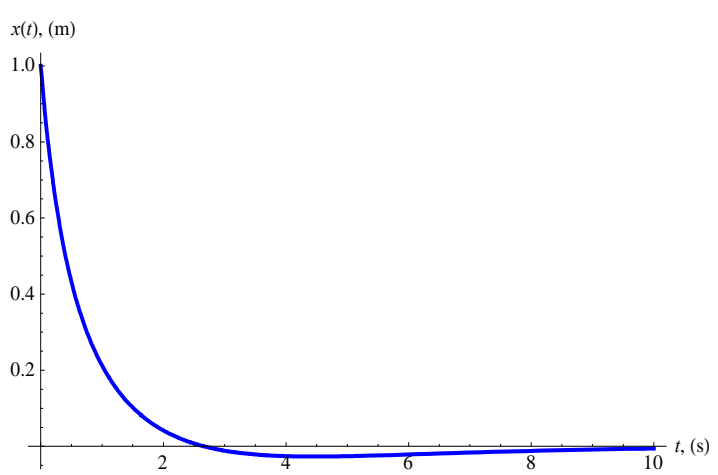

Fig. 2. The exact solution with $\beta=2.5, x_{0}=1$ and $\dot{x}_{0}=-2$

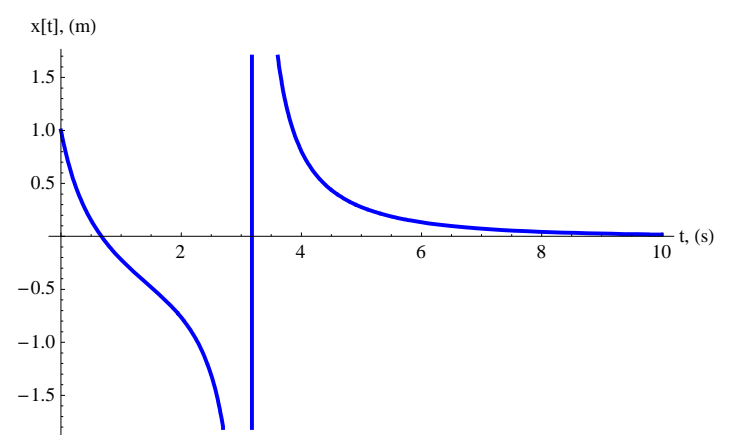

Fig. 3. The exact solution with $\beta=2.5, x_{0}=1$ and $\dot{x}_{0}=-3$

\subsection{Case $m=2$}

Parametric conditions (7) and (13) providing integrability of Eq. (1) in this case are following

$$
\delta=\frac{4}{\beta}, \quad \mu=-\frac{3}{\beta^{2}},
$$

and the original equation (1) has of the form

$$
\ddot{x}+\left(\frac{4}{\beta}+\beta x^{2}\right) \dot{x}+\frac{3}{\beta^{2}} x+x^{3}=0 .
$$

The exact solution is represented in parametric from Eqs. (26) and (28) with $m=2$, $a=\beta$ and $c=\frac{3}{\beta}$.

Suppose that input data are given $\beta=2.5$ and initial values $x_{0}=1, \dot{x}_{0}=0.4$. Integral constants are calculated by Eqs. (31) and (32) as follows

$$
D_{2}=1.96, \quad D_{2}^{1 / 3}=1.25146, \quad \gamma=1.58142 .
$$


The exact parametric solutions of the given equation are represented by Eqs. (26), (28) and (29). The results are illustrated in Figs. 4-6.

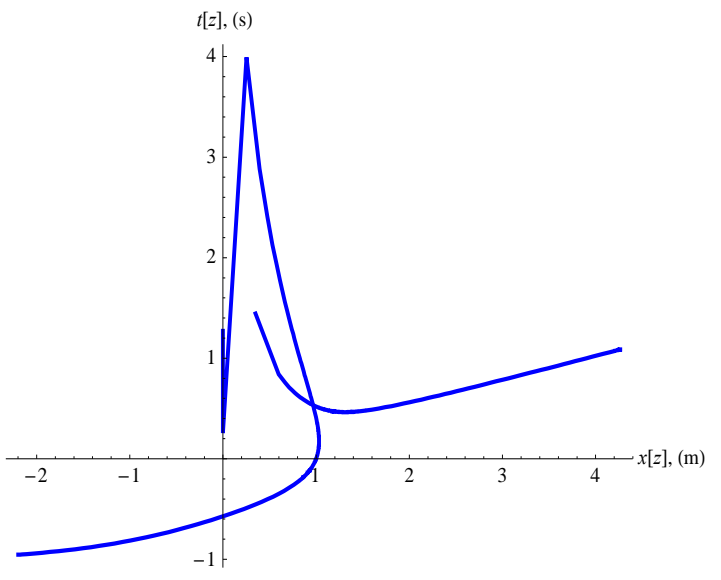

Fig. 4. Graphic of parametric solution $x(z)-$ $t(z)$ with $z(-1.5 ; 1.25145)$ and $z(1.25147 ; 3.8)$

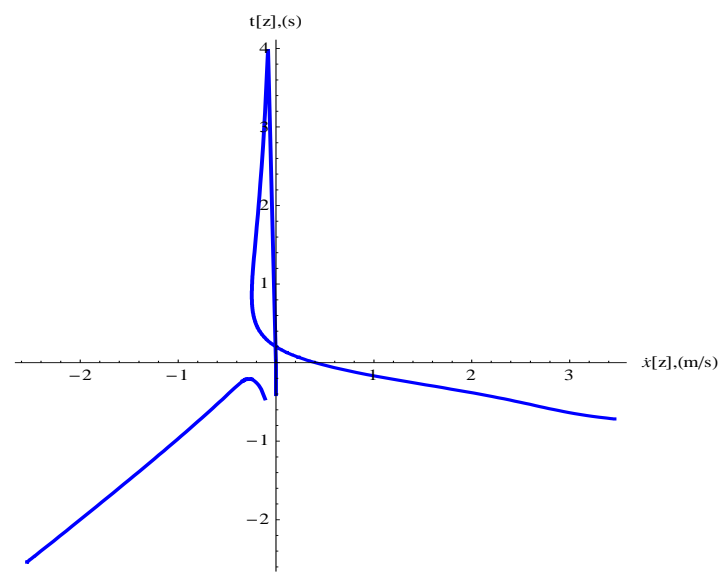

Fig. 5. Graphic of parametric solution for velocity $\dot{x}(z)-t(z)$ with $z(-0.4 ; 1.25145)$ and $z(1.25147 ; 1.7)$

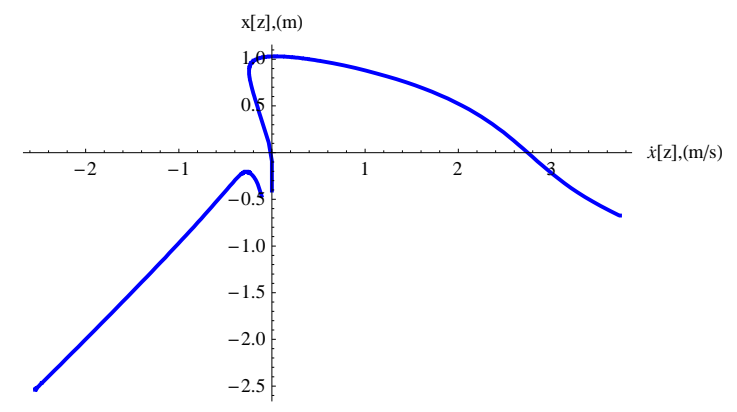

Fig. 6. Graphic of parametric phase curve in the phase plane $\dot{x}(z)-x(z)$ with $z(-0.5 ; 1.25145)$ and $z(1.25147 ; 1.7)$

As can see that the parametric solution has a discontinuous point corresponding to $z=1.25146$. The parametric solution for velocity and phase curve have a discontinuous point also at $z=1.25146$.

5.3. Case $m=3$

The parametric conditions in this case reduced from (7) and (13) are

$$
\delta=\frac{5}{\beta}, \quad \mu=-\frac{4}{\beta^{2}},
$$

and the original equation (1) has of the form

$$
\ddot{x}+\left(\frac{5}{\beta}+\beta x^{3}\right) \dot{x}+\frac{4}{\beta^{2}} x+x^{4}=0 .
$$


The parametric exact solution is described by Eqs. (26) and (28) with $m=3, a=\beta$ and $c=\frac{4}{\beta}$.

The input data are supposed $\beta=2$ and given initial values $x_{0}=1, \dot{x}_{0}=0.4$. Integral constants $D_{2}, \gamma$ are valued as

$$
D_{2}=2.8, \quad \gamma=2.16835
$$

The parametric exact solutions defined by Eqs. (26), (28) and (29) are illustrated in Figs. 7-9.

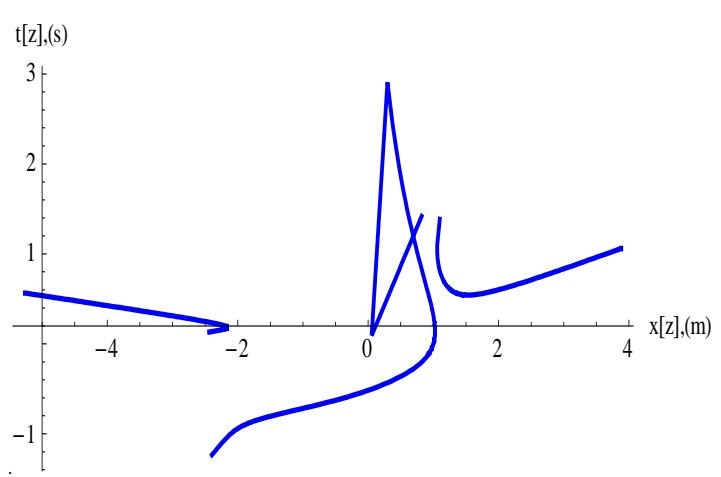

Fig. 7. Graphic of parametric solution $x(z)-$ $t(z)$ with $z(-3.5 ;-1.2936), z(-1.2935 ; 1.2935)$ and $z(1.2936 ; 3.5)$

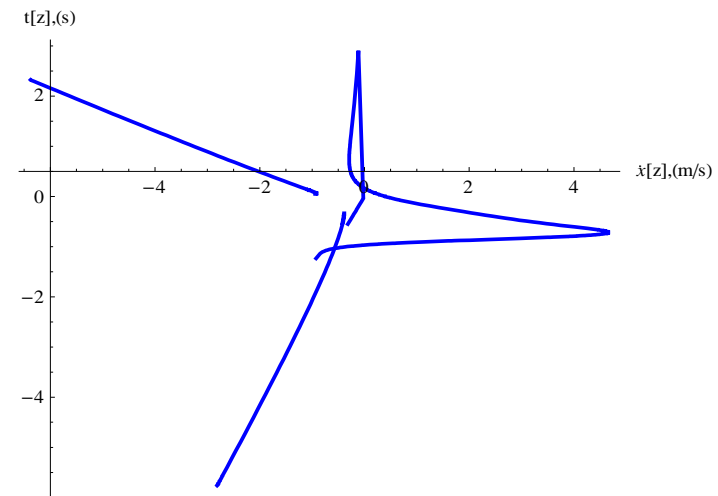

Fig. 8. Graphic of parametric solution for velocity $\dot{x}(z)-t(z)$ with $z(-1.48 ;-1.2936)$, $z(-1.2935 ; 1.2935)$ and $z(1.2936 ; 1.8)$

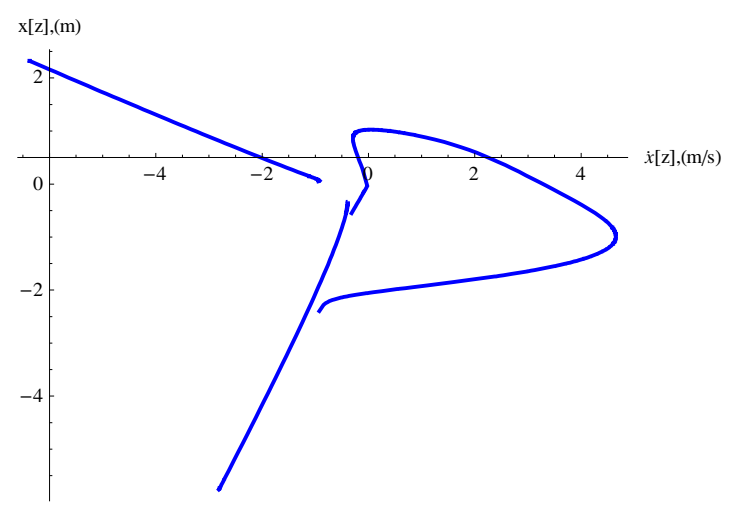

Fig. 9. Graphic of parametric phase curve in the phase plane $\dot{x}(z)-x(z)$ with $z(-1.48 ;-1.2936)$, $z(-1.2935 ; 1.2935)$ and $z(1.2936 ; 1.8)$

It is observed that the parametric solution has two discontinuous points corresponding to $z= \pm 1.29357$ and the velocity and the phase curve also have two discontinuous points at the same $z= \pm 1.29357$. 
5.4. Case $m=\frac{2}{3}$

The parametric conditions (7) and (13) are reduced to

$$
\delta=\frac{8}{3 \beta}, \quad \mu=-\frac{5}{3 \beta^{2}},
$$

and the original equation (1) in this case

$$
\ddot{x}+\left(\frac{8}{3 \beta}+\beta x^{2 / 3}\right) \dot{x}+\frac{5}{3 \beta^{2}} x+x^{5 / 3}=0,
$$

gives an exact solution demonstrated by Eqs. (26) and (28) with $m=\frac{2}{3}, a=\beta$ and $c=\frac{5}{3 \beta}$.

For example the input data are given as

$$
\beta=1, \quad m=2 / 3, \quad x_{0}=1, \quad \dot{x}_{0}=0.4 \text {. }
$$

Integral constants are valued

$$
D_{2}=3.33333, \quad D_{2}^{3 / 5}=2.05934, \quad \gamma=1.37973 \text {. }
$$

The parametric solutions determined by Eqs. (26), (28) and (29) are demonstrated in Figs. 10-12.

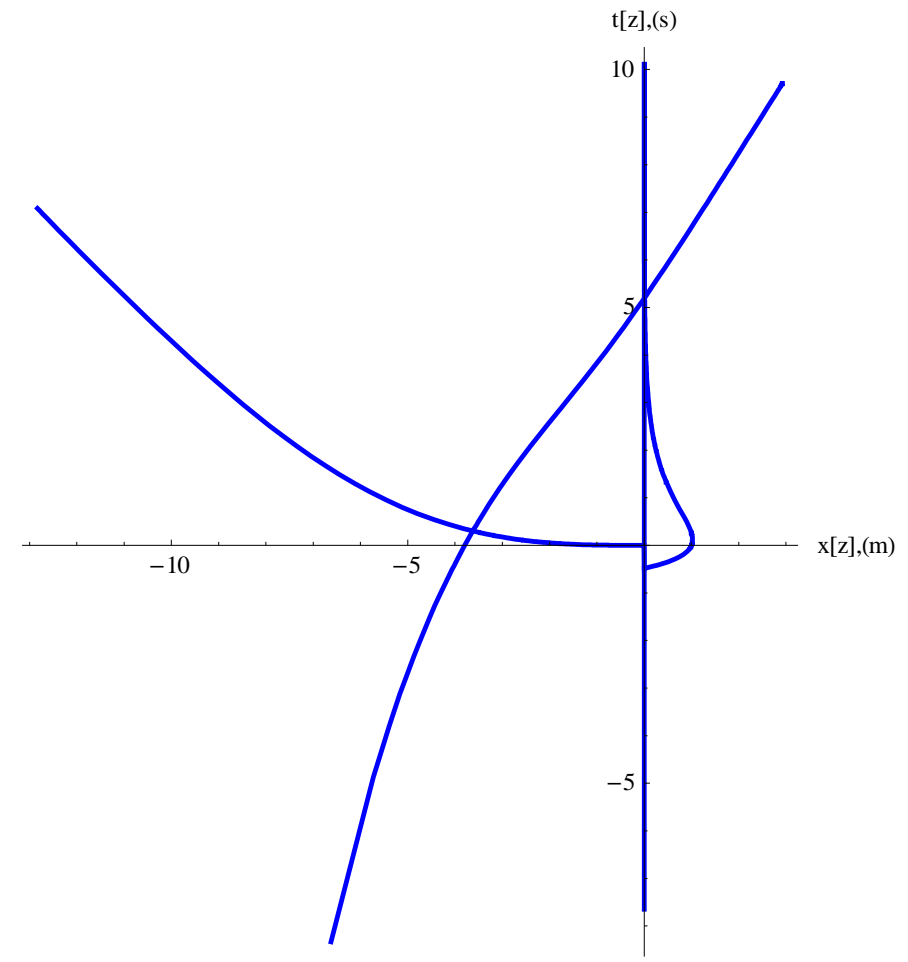

Fig. 10. Graphic of parametric solution $x(z)-t(z)$ with $z(-2 ; 0), z(0 ; 2.059)$ and $z(2.0595 ; 3)$ 


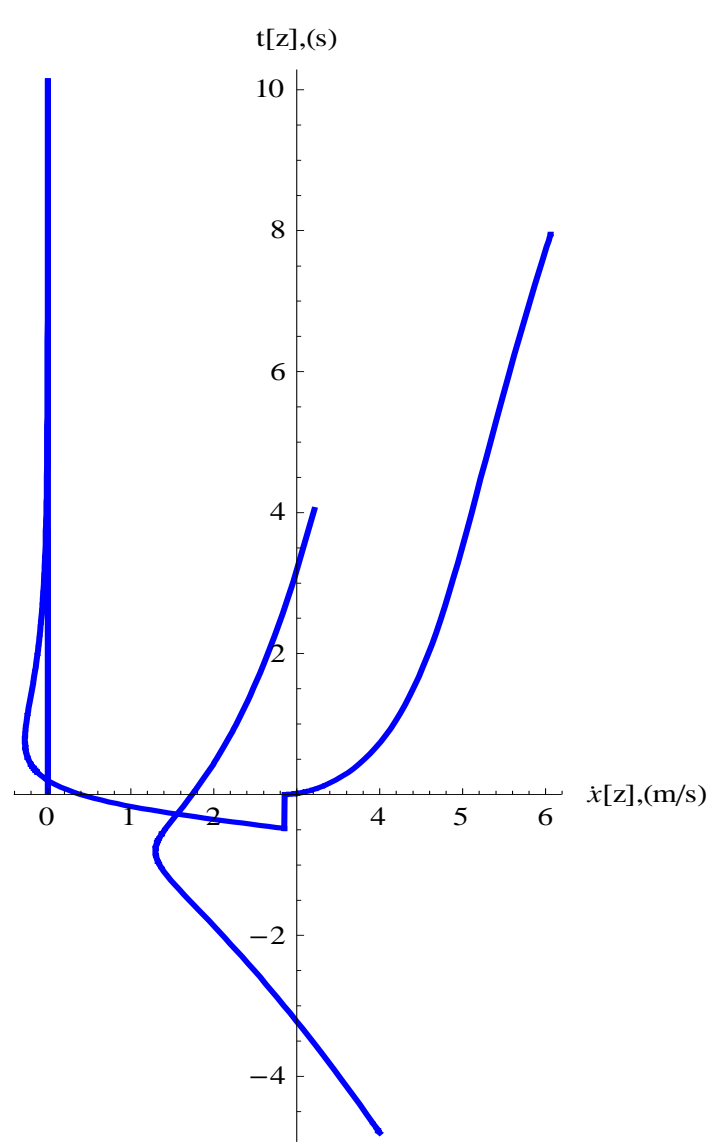

Fig. 11. Graphic of parametric solution for velocity $\dot{x}(z)-t(z)$ with $z(-2 ; 0), z(0 ; 2.059)$ and $z(2.0595 ; 3)$

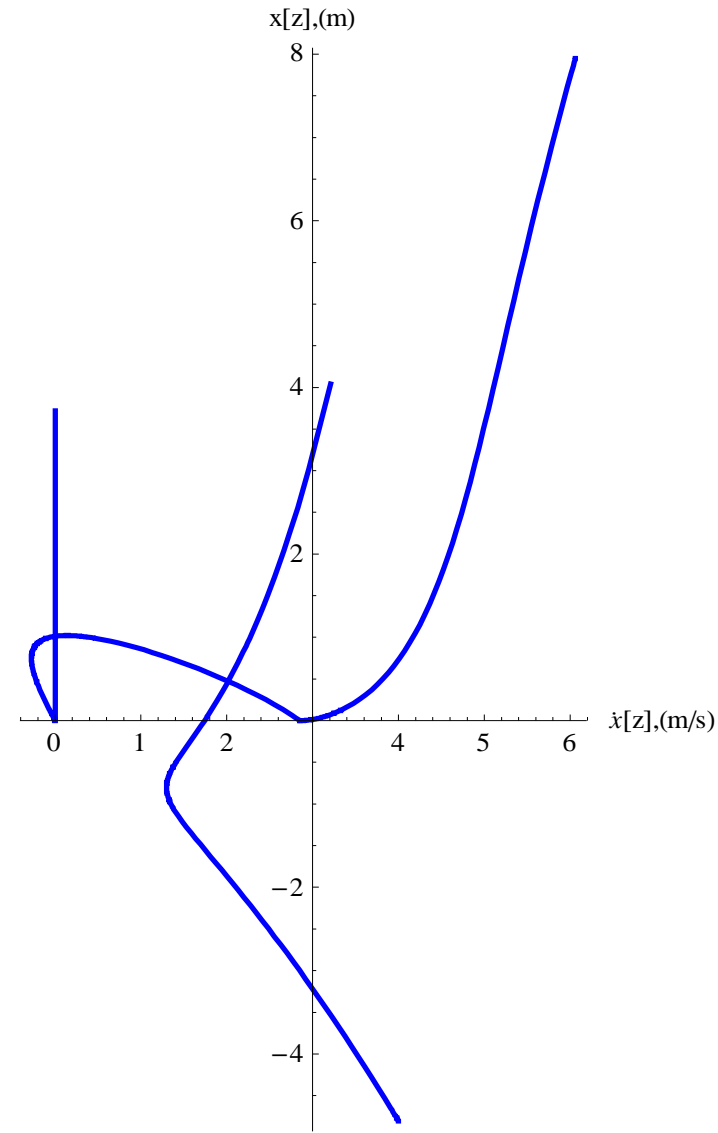

Fig. 12. Graphic of parametric phase curve in the phase plane $\dot{x}(z)-x(z)$ with $z(-2 ; 0)$, $z(0 ; 2.059)$ and $z(2.0595 ; 3)$

As can see that, the parametric solutions have two discontinuous points corresponding to $z=0$ and $z=2.05934$.

Remark. In the considered cases 5.2-5.4, the solution can be expressed by hypergeometric function of involved parameters. This function can be developed by power series which may be converged in or out of unit circle depending on the parameter $z$. The points where the functions are not converged represent discontinuous points as indicated in from Fig. 3 to Fig. 12.

\section{Discussion}

The closed formed solution can be expressed through a hypergeometric function of involved parameters, see (26), (27), (28). This has traditionally been expressed as integral functions.

In case 1, parametric condition and exact solution to the Riccati equation can be obtained when $m=1$. In case 4 , parametric condition and exact solution when $m$ is 
not an integer number can also be found. Note that those are traditionally regarded as equations without exact solutions.

An identical solution in comparison with results from the symmetry Lie method when $m=2$, see (16) and (17).

\section{CONCLUSIONS}

A method for finding parametric condition and exact solution to the Duffing-Van der Pol class of equations has been proposed.

Exact solutions and respective parametric conditions to the Duffing-Van der Pol class of equations can be found with any order of $m$, with $m$ being a real number. The proposed methodology is proved to be more general than the pioneered by the Parelle and Singer method and the symmetry Lie method.

A method to reduce the order of the original differential equation is proposed. The solution procedure for an exact solution can therefore be simplified.

Parametric condition and exact solution to the Riccati equation can be obtained when $m=1$. Parametric condition and exact solution when $m$ is not an integer number can also be found. Note that those are traditionally regarded as equations without exact solutions.

With closed formed solution, observation on solution characteristic such as discontinuity, bifurcation, and asymptotic can conveniently be made.

\section{REFERENCES}

[1] M. J. Prelle and M. F. Singer. Elementary first integrals of differential equations. Transactions of the American Mathematical Society, 279, (1), (1983), pp. 215-229. https://doi.org/10.1090/s0002-9947-1983-0704611-x.

[2] L. G. S. Duarte, S. E. S. Duarte, L. A. C. P. da Mota, and J. E. F. Skea. Solving second-order ordinary differential equations by extending the Prelle-Singer method. Journal of Physics A: Mathematical and General, 34, (14), (2001), pp. 3015-3024. https://doi.org/10.1088/0305$4470 / 34 / 14 / 308$.

[3] V. K. Chandrasekar, M. Senthilvelan, and M. Lakshmanan. On the complete integrability and linearization of certain second-order nonlinear ordinary differential equations. In Proceedings of the Royal Society of London A: Mathematical, Physical and Engineering Sciences, Vol. 461. The Royal Society, (2005), pp. 2451-2477. https://doi.org/10.1098/rspa.2005.1465.

[4] M. S. Velan and H. Lakshmanan. Lie symmetries and infinite-dimensional Lie algebras of certain nonlinear dissipative systems. Journal of Physics A: Mathematical and General, 28, (7), (1995), pp. 1929-1942. https://doi.org/10.1088/0305-4470/28/7/015.

[5] V. K. Chandrasekar, M. Senthilvelan, and M. Lakshmanan. New aspects of integrability of force-free Duffing-van der Pol oscillator and related nonlinear systems. Journal of Physics A: Mathematical and General, 37, (16), (2004), pp. 4527-4534. https://doi.org/10.1088/0305$4470 / 37 / 16 / 004$.

[6] D. W. Jordan and P. Smith. Nonlinear ordinary differential equations: an introduction to dynamical systems. Oxford University Press, New York, (2007).

[7] M. Lakshmanan and S. Rajaseekar. Nonlinear dynamics: integrability, chaos and patterns. Springer Science \& Business Media, (2012).

[8] G. Gao and Z. Feng. First integrals for the Duffing-van der Pol type oscillator. Electronic Journal of Differential Equations, 19, (2010), pp. 123-133. 
[9] D. H. Bich and N. D. Bich. A coupling successive approximation method for solving Duffing equation and its application. Vietnam Journal of Mechanics, 36, (2), (2014), pp. 77-93. https://doi.org/10.15625/0866-7136/36/2/4095.

[10] Wolfram Mathematica Tutorial Collection. Mathematics and Algorithms, Wolfram Research, Inc., United States of America, (2008). 